\title{
Attachment Centrality for Weighted Graphs
}

\author{
Jadwiga Sosnowska, Oskar Skibski \\ University of Warsaw, Poland \\ \{j.sosnowska,o.skibski\}@mimuw.edu.pl
}

\begin{abstract}
Measuring how central nodes are in terms of connecting a network has recently received increasing attention in the literature. While a few dedicated centrality measures have been proposed, Skibski et al. [2016] showed that the Attachment Centrality is the only one that satisfies certain natural axioms desirable for connectivity. Unfortunately, the Attachment Centrality is defined only for unweighted graphs which makes this measure ill-fitted for various applications. For instance, covert networks are typically weighted, where the weights carry additional intelligence available about criminals or terrorists and the links between them. To analyse such settings, in this paper we extend the Attachment Centrality to node-weighted and edgeweighted graphs. By an axiomatic analysis, we show that the Attachment Centrality is closely related to the Degree Centrality in weighted graphs.
\end{abstract}

\section{Introduction}

Centrality analysis belongs to fundamental research problems in network analysis. Its aim is to quantify the importance of nodes in a network according to some criteria that typically depend on the application at hand [Brandes and Erlebach, 2005]. For instance, in the transportation network we may look for nodes which are closest on average to all other nodes in the network [Tarkowski et al., 2016] or which are positioned on most shortest paths between nodes [Derrible, 2012].

One particular such criterion that has recently attracted a lot of attention in the literature is connectivity. Intuitively, this means that we are interested in nodes that are essential to keeping the network together. At a first glance, it may seem that we should simply look for nodes with the highest degree, i.e., the number of incident edges. However, a need for more advanced measures of connectivity has been advocated in a number of different areas, spanning from evaluation of the importance of genes in gene interaction networks [Moretti et al., 2010] and assessing the impact of failure in monetary flows networks [Belau, 2014] to social network analysis [Narayanam et al., 2014] and the analysis of covert networks [Lindelauf et al., 2013].
Most centrality measures dedicated to connectivity were constructed in various contexts of particular applications. Unfortunately, such results are difficult to generalize especially given that they were evaluated solely based on experiments with some real-life and synthetic networks. Only recently, Skibski et al. [2016] took a more general, axiomatic approach and showed that there exists a unique centrality measure that satisfies some natural axioms that may be considered desirable for connectivity.

This new centrality, called Attachment Centrality, is defined for unweighted graphs, i.e., graphs whose nodes and edges have not been assigned any weights and each two nodes or two edges are equally important. This assumption, however, does not hold in many applications [Bagler, 2008; Nepusz et al., 2012; Palla et al., 2007]. One example is covert network analysis - a setting that has recently attracted considerable attention in the literature. In these networks, weights of nodes and edges may carry additional intelligence available about criminals or terrorists and the links between them. For instance, weights of edges are used to represent frequency of interaction [Koschade, 2006] while weights of nodes to represent individual evaluations of terrorists, based on known skills and prior activities [Lindelauf et al., 2013].

Unfortunately, neither is the Attachment Centrality in its current form able to incorporate such data nor is it clear how this centrality can be adapted to do so. Specifically, the axiomatization of the Attachment Centrality relies on Normalization. This axiom states both the minimum and maximum bounds on the values of centrality and specifies the values for the center of a star and for the isolated nodes. However, in weighted graphs, centrality is unbounded and the value of the center of a star is far from obvious.

In this paper, we propose the first extension of the Attachment Centrality to node- and edge-weighted graphs. We begin by developing a new axiomatization of the Attachment Centrality for unweighted graphs. It is built upon two new axioms - One-Edge Normalization and Additivity - and two already proposed in the literature: Balanced Contributions [Myerson, 1980]; and Gain-loss [Skibski et al., 2016]. Next, we extend this axiomatization to node-weighted graphs, and finally to node- and edge-weighted graphs and propose the corresponding formulas for the Attachment Centrality. As a reference, we build this axiomatization in a close relation to the Degree Centrality [Freeman, 1979]. 


\section{Preliminaries}

This section provides the necessary background and notation.

Graph theory: A (undirected) graph is a tuple, $(V, E, \omega, \rho)$, where $V$ is the set of nodes, $E$ is the set of edges, and $\omega, \rho$ are weight functions (see below). Given $V$, the set of all possible graphs is denoted by $\mathcal{G}^{V}$. For $G=(V, E, \omega, \rho)$ and $E^{\prime} \subseteq E$ we use shorthand notations $G+E^{\prime}$ (and $G-E^{\prime}$ ) to denote the graph obtained by adding $E^{\prime}$ to $E$ (removing $E^{\prime}$ from $E$ ).

An edge $\{v, u\} \in E$ is said to be incident to nodes $v$ and $u$, and $u$ is said to be a neighbour of $v$. For node $v \in V$, the set of its neighbours is denoted by $N_{G}(v)$ and the set of its (incident) edges is denoted by $\Gamma_{G}(v)$. Formally: $N_{G}(v)=$ $\{u \in V:\{v, u\} \in E\}$ and $\Gamma_{G}(v)=\{\{v, u\} \in E: u \in V\}$. If a node, $v$, has no neighbours, i.e., $N_{G}(v)=\Gamma_{G}(v)=\emptyset$, we say that $v$ is isolated. If a node has exactly one neighbour, we call it a leaf.

A path, $p=\left(v_{1}, \ldots, v_{k}\right)$, is a sequence of nodes in which there exists an edge between every two consecutive nodes, i.e., $\left\{v_{i}, v_{i+1}\right\} \in E, \forall i \in\{1, \ldots, k-1\}$. Nodes $v, u \in V$ are said to be connected if there exists a path between them. A graph $G$ is connected if every two nodes in it are connected. For any subset of nodes, $S \subseteq V$, the subgraph with nodes in $S$ and all edges that both ends belong to subset $S$ is called the subgraph induced by $S$. Formally, $G[S]=(S, E[S], \omega, \rho)$ and $E[S]=\{\{v, u\} \in E: v, u \in S\}$. For a disconnected graph, $G$, we denote by $K(G)$ the partition of $V$ into disjoint sets of nodes, called connected components, that each induces a maximal connected subgraph in $G$. Finally, we denote by $K_{v}(G)$ the connected component containing $v$ in $G$.

A forest is a graph without cycles, i.e., a graph such that there exists at most one path between every two nodes. A connected forest is called a tree. A star is a tree in which there exists a node, $v$, called the center of a star, that is connected by an edge with every other node: $E=\{\{v, u\}: u \in V \backslash\{v\}\}$.

Graph weights: Function $\omega: V \rightarrow \mathbb{R}^{+}$is a node-weight function that assigns to every node in $V$ its weight. Function $\rho: E \rightarrow \mathbb{R}^{+}$is an edge-weight function that assigns to every edge in $E$ its weight. A function that assigns 1 to every argument is denoted by 1 . A graph is unweighted if $\omega=1$ and $\rho=1$. Otherwise, if $\omega \neq 1$ and $\rho=1$, then graph is called a node-weighted graph. If $\omega \neq \mathbf{1}$ and $\rho \neq 1$, a graph is called a node- and edge-weighted graph.

For a function, $f: X \rightarrow \mathbb{R}^{+}, x \in X$, and $\alpha \in \mathbb{R}^{+}$we write $f_{x}^{\alpha}$ to denote the function obtained by changing the value of $x$ in $f$ to $\alpha: f_{x}^{\alpha}(x)=\alpha$ and $f_{x}^{\alpha}(y)=f(y)$ for every $y \in X \backslash$ $\{x\}$. Moreover, we use $f_{-x}$ to denote the function obtained by removing argument $x$ from the domain of $f$.

Cooperative games: A (cooperative) game is a pair $(V, f)$, where $V$ is the set of players and $f: 2^{V} \rightarrow \mathbb{R}$ is the characteristic function. The characteristic function assigns to each subset of players, called a coalition, a real number, called the value of a coalition, where $f(\emptyset)=0$. Typically, we will refer to the game simply by its characteristic function $f$. A value of a game, $\varphi:\left(2^{V} \rightarrow \mathbb{R}\right) \rightarrow \mathbb{R}^{V}$, is a function that assigns a payoff to each player $v \in V$. This payoff can be interpreted as the player's importance in the game. One of the most important such values was proposed by Shapley [1953b]. The
Shapley value of player $v$ in game $f$, denoted by $S V_{v}(f)$, is defined as:

$$
S V_{v}(f)=\frac{1}{|V| !} \sum_{\pi \in \Omega(V)}\left(f\left(S_{v}^{\pi}\right)-f\left(S_{v}^{\pi} \backslash\{v\}\right)\right)
$$

where $\Omega(V)$ is the set of all permutations of $V$, i.e., bijections $\pi: V \rightarrow\{1, \ldots,|V|\}$, and $S_{v}^{\pi}=\{u \in V: \pi(u) \leq \pi(v)\}$ is the set of players at the position of $v$ or earlier in $\pi$. Importantly, the Shapley value satisfies Additivity $\left(\varphi(f)+\varphi\left(f^{\prime}\right)=\right.$ $\left.\varphi\left(f+f^{\prime}\right)\right)$ and Efficiency $\left(\sum_{v \in V} \varphi_{v}(f)=f(V)\right)$.

Myerson [1977] extended the Shapley value to the model in which the cooperation of players is restricted by a communication graph. The Myerson value for player $v$ in game $f$, denoted by $M V_{v}(f, G)$, is defined as the Shapley value of the restricted game $f / G: M V_{v}(f, G)=S V_{v}(f / G)$, where $f / G(S)=\sum_{C \in K(G[S])} f(C)$ for every $S \subseteq V$. Myerson proved that the Myerson value is the only value that satisfies Component Efficiency and Fairness. The former axiom states that for every connected component $C$ the sum of values of nodes in $C$ equals $f(C)$. The later one requires that addition of an edge equally affects the two nodes connected by it. Later on, Myerson [1980] proposed Balanced Contributions (see Section 3) and proved that it implies Fairness.

Centrality measures: A centrality measure, $F: \mathcal{G}^{V} \rightarrow \mathbb{R}^{V}$, is a function that assigns to every node a number reflecting its importance. Thus, it plays the same role as a value of a game.

The one of the most well-known centrality measures is the Degree Centrality. For an unweighted graph, the Degree Centrality rates every node taking into consideration only a number of its neighbours, i.e.: $D_{v}(V, E, \mathbf{1}, \mathbf{1})=\left|N_{G}(v)\right|$. For a weighted graph, the Degree Centrality extends to:

$$
W D_{v}(V, E, \omega, \rho)=\sum_{u \in N_{G}(v)} \rho(\{v, u\}) \cdot \omega(u) .
$$

This definition follows [Opsahl et al., 2010; Heitzig et al., 2010]. We will refer to this centrality measure as the Weighted Degree Centrality.

Skibski et al. [2016] proposed the Attachment Centrality as a dedicated measure of connectivity.

Definition 1. The Attachment Centrality for an unweighted graph $G=(V, E, \mathbf{1}, \mathbf{1})$ is defined as follows:

$$
A_{v}(G)=S V_{v}\left(f_{G}^{*}\right)
$$

where $f_{G}^{*}(S)=2(|S|-|K(G[S])|)$ for every $S \subseteq V$.

Alternatively, $A_{v}(G)=M V\left(f^{*}, G\right)$ for $f^{*}(S)=2(|S|-1)$ for every $S \subseteq V$. Skibski et al. proved that Attachment Centrality is the unique centrality that satisfies Myerson's Fairness, Normalization, Locality, and Gain-loss. Normalization states that $F_{v}(G) \in[0,|V|-1]$ and $F_{v}(G)=0$, when $v$ is isolated in $G$, and $F_{v}(G)=|V|-1$, when $G$ is a star, the center of which is $v$. Locality states that the centrality of a node depends solely on the connected component the node belongs to (i.e., removing other components does not affect the centrality of a node). Gain-loss will be described later.

Our goal in this paper is to extend Attachment Centrality to node-weighted and edge-weighted graphs. To this end, we first propose a new axiomatization of Attachment Centrality for unweighted graphs. Upon this result we will later on build an axiomatization of Attachment Centrality for weighted graphs. 


\section{Unweighted Graphs}

In this section, we propose a new axiomatization of the Attachment Centrality that does not rely on Normalization. Our new axiomatization involves Balanced Contributions by Myerson [1980], Gain-loss by Skibski et al. [2016] and two new axioms - Additivity and One-Edge Normalization. We follow the idea of Skibski et al. and build an axiomatization in a close relation to the Degree Centrality. In fact, all above axioms except Gain-loss are satisfied by the Degree Centrality, and we prove that adding Star-Max and Monotonicity leads to an axiomatization of the Degree Centrality.

We begin by introducing two new axioms. Firstly, we replace Normalization with much simpler axiom, called One-Edge Normalization. More in detail, One-Edge Normalization states that if there is only one edge in the graph, then both adjacent nodes have centralities equal to one.

One-Edge Normalization: For every $v, u \in V$,

$$
F_{v}(V,\{\{v, u\}\}, \mathbf{1}, \mathbf{1})=1 .
$$

Our second axiom, Additivity, states that the centrality of a node that connects disjoint parts of the graph is simply the sum of its centralities computed for each part separately.

Additivity: For every unweighted graph $G=(V, E, \mathbf{1}, \mathbf{1})$, node $v \in V$ and sets $S, T \subseteq V$ such that $S \cap T=\{v\}$, $F_{v}(V, E[S], \mathbf{1}, \mathbf{1})+F_{v}(V, E[T], \mathbf{1}, \mathbf{1})=F_{v}(V, E[S] \cup E[T], \mathbf{1}, \mathbf{1})$.

The third axiom, Balanced Contributions [Myerson, 1980], states that removing edges of $v$ affects the centrality of $u$ in the same way as removing edges of $u$ affects the centrality of $v$.

Balanced Contributions: For every unweighted graph $G=(V, E, \mathbf{1}, \mathbf{1})$ and every two nodes $v, u \in V$,

$$
F_{v}(G)-F_{v}\left(G-\Gamma_{G}(u)\right)=F_{u}(G)-F_{u}\left(G-\Gamma_{G}(v)\right) .
$$

Finally, we present Gain-loss, first proposed by Skibski et al. [2016]. It states that the sum of centralities of a graph does not change when we add or remove edges, as far as the set of connected components stays the same. ${ }^{1}$

Gain-loss: For every unweighted graph $G=(V, E, \mathbf{1}, \mathbf{1})$ and $v, u \in C \in K(G)$,

$$
\sum_{w \in V} F_{w}(G)=\sum_{w \in V} F_{w}(G+\{\{v, u\}\}) .
$$

We will use Gain-loss to axiomatize the Attachment Centrality. For comparison, let us also axiomatize the Degree Centrality. Here, we will use Monotonicity and Star-Max. Monotonicity was proposed by Skibski $e t$ al. and states that adding an edge does not decrease the centrality of any node.

Monotonicity: For every unweighted graph $G=$ $(V, E, \mathbf{1}, \mathbf{1})$ and $v, u, w \in V$,

$$
F_{v}(G) \leq F_{v}(G+\{\{u, w\}\}) .
$$

The last axiom, Star-Max, inspired by Normalization, states only that a centrality of a node is maximized when it is the center of a star, but it does not specify a value.

\footnotetext{
${ }^{1}$ This is a slight modification of the original axiom that considers only adding edges to a connected graph, while we consider adding edges inside a connected component of possibly disconnected graph.
}

Star-Max: For every unweighted graph $G=(V, E, \mathbf{1}, \mathbf{1})$ and $v \in V$,

$$
F_{v}(G) \leq F_{v}(V,\{\{v, u\}: u \in V\}, \mathbf{1}, \mathbf{1}) .
$$

In the following theorem, we show that Balanced Contributions, Additivity, and One-Edge Normalization imply that the centrality of a node in a tree equals its number of neighbours. In particular, the centrality of an isolated node is 0 .

Theorem 1. Let $G=(V, E, \mathbf{1}, \mathbf{1})$ be an unweighted graph and $v \in V$. If a centrality measure, $F$, satisfies Balanced Contributions, Additivity, and One-Edge Normalization and $K_{v}(G)$ is a tree, then $F_{v}(G)=\left|N_{G}(v)\right|$.

The proof is analogous to the proof of Theorem 4 for weighted graphs, so we omit details. We are now ready to state the main theorems of this section that characterize the Degree and Attachment Centralities in unweighted graphs.

Theorem 2. If a centrality measure, $F$, satisfies Balanced Contributions, Additivity, One-Edge Normalization, StarMax and Monotonicity, then for every unweighted graph $G=(V, E, \mathbf{1}, \mathbf{1})$ and $v \in V$ it is equal to the Degree Centrality: $F_{v}(G)=D_{v}(G)$.

Sketch of Proof. The proof is based on induction on the number of edges. From Monotonicity and Theorem 1 it can be shown that $F_{v}(G) \geq D_{v}(G)$. To prove that $F_{v}(G) \leq D_{v}(G)$ we use graph $G^{\prime}$ where $V \backslash\{v\}$ forms a clique and prove that $F_{v}\left(G^{\prime}\right)=D_{v}\left(G^{\prime}\right)$ using the inductive assumption. Due to space constraints we omit details.

Theorem 3. If a centrality measure, $F$, satisfies Balanced Contributions, Additivity, One-Edge Normalization and Gain-loss, then for every unweighted graph $G=(V, E, \mathbf{1}, \mathbf{1})$ and $v \in V$ it is equal to the Attachment Centrality: $F_{v}(G)=A_{v}(G)$.

Proof. Let $G=(V, E, \mathbf{1}, \mathbf{1})$ be an unweighted graph. Fix $v \in V$. First, we will show that a centrality of node $v$ does not depend on edges from other connected components in graph $G$. From Additivity we get $F_{v}(V, E, \mathbf{1}, \mathbf{1})=$ $F_{v}\left(V, E\left[K_{v}(G)\right], \mathbf{1}, \mathbf{1}\right)+F_{v}\left(V, E\left[V \backslash K_{v}(G)\right], \mathbf{1}, \mathbf{1}\right)$. Node $v$ in the later graph is isolated and from Theorem 1 its centrality equals 0 . Thus, we get $F_{v}(V, E, \mathbf{1}, \mathbf{1})=$ $F_{v}\left(V, E\left[K_{v}(G)\right], \mathbf{1}, \mathbf{1}\right)$.

Let us compute the sum of centralities of nodes in component $K_{v}(G)$. To this end, let $G^{\prime}=\left(V, E^{\prime}, \mathbf{1}, \mathbf{1}\right)$ be a graph obtained from $G$ by removing edges in the component of $v$ so that $K_{v}\left(G^{\prime}\right)$ is a tree. From Theorem 1, we know that for every $u \in K_{v}(G)$ we have $F_{u}\left(G^{\prime}\right)=\left|N_{G^{\prime}}(u)\right|$. Summing over all nodes $u \in K_{v}(G)$ and using Gain-loss we get that

$$
\sum_{u \in K_{v}(G)} F_{u}(G)=\sum_{u \in K_{v}(G)} F_{u}\left(G^{\prime}\right)=2 \cdot\left(\left|K_{v}(G)\right|-1\right) .
$$

Finally, from [Skibski et al., 2017, Lemma 5] we get that there exists a unique centrality that satisfies Fairness and has specific sum of centralities for every component. Since Balanced Contributions implies Fairness, from Equation (2) we get that there exists at most one centrality that satisfies axioms from the statement of Theorem 3. It is easy to check that the Attachment Centrality satisfies these axioms, so $F_{v}(G)=$ $A_{v}(G)$, which concludes the proof of Theorem 3 . 


\section{Node-Weighted Graphs}

In this section, we extend the Attachment Centrality to nodeweighted graphs.

Following the analysis from Section 3, our starting point is the analysis of axioms in weighted trees.

Example 1. Consider a node-weighted graph with one edge $\{v, u\}: G=(V,\{\{v, u\}\}, \omega, \mathbf{1})$. For this graph we have:

$$
W D_{v}(G)=\omega(u) \text { and } W D_{u}(G)=\omega(v) .
$$

Clearly, One-Edge Normalization is not satisfied for weighted graphs. Moreover, Balanced Contributions needs to be revisited. Observe that the profit from adding edge $\{v, u\}$ is higher for a node with a lower weight. Thus, in case of the Weighted Degree Centrality, the profit is inversely proportional to the weight of a node.

This example leads to the generalization of One-Edge Normalization and Balanced Contributions.

Weighted One-Edge Normalization: For every $v, u \in V$,

$$
F_{v}(V,\{\{v, u\}\}, \omega, \mathbf{1})=\omega(u) .
$$

Let us introduce Inverse Weighted Balanced Contributions. This is a new axiom which states that for every two nodes $v, u \in V$, removing edges of $v$ affects the centrality of $u$ multiplied by its weight in the same way as removing edges of $u$ affects the centrality of $v$ multiplied by its weight.

Inverse Weighted Balanced Contributions: For every node-weighted $G=(V, E, \omega, \mathbf{1})$ and every $v, u \in V$,

$$
\frac{F_{v}(G)-F_{v}\left(G-\Gamma_{G}(u)\right)}{\omega(u)}=\frac{F_{u}(G)-F_{u}\left(G-\Gamma_{G}(v)\right)}{\omega(v)} .
$$

The name of Inverse Weighted Balanced Contributions comes from the fact that the weight of a node $v$ is inversely correlated with the change in its centrality.

Finally, Additivity, Star-Max, and Monotonicity naturally translate to node-weighted graphs by replacing 1 with an arbitrary $\omega$ function in the graph definition. Due to space constraints we omit details. Now, Inverse Weighted Balanced Contributions, Additivity and Weighted One-Edge Normalization uniquely characterize the centrality for trees.

Theorem 4. Let $G=(V, E, \omega, \mathbf{1})$ be a node-weighted graph and $v \in V$. If a centrality measure, $F$, satisfies Inverse Weighted Balanced Contributions, Additivity, and Weighted One-Edge Normalization and $K_{v}(G)$ is a tree, then $F_{v}(G)=$ $\sum_{u \in N_{G}(v)} \omega(u)$.

Proof. Assume $F$ satisfies Inverse Weighted Balanced Contributions, Additivity and Weighted One-Edge Normalization and fix a node-weighted graph $G=(V, E, \omega, \mathbf{1})$ and $v \in V$. We divide the proof in three parts:

(a) if $N_{G}(v)=\emptyset(v$ is isolated $)$, then $F_{v}(G)=0$;

(b) if $N_{G}(v)=\{u\}$ ( $v$ is a leaf), then $F_{v}(G)=\omega(u)$;

(c) if $\left|N_{G}(v)\right|=k$ and $\left|K\left(G-\Gamma_{G}(v)\right)\right|-|K(G)|=k$, then $F_{v}(G)=\sum_{u \in N_{G}(v)} \omega(u)$.

(a) Since $\Gamma_{G}(v)=\emptyset$, from Inverse Weighted Balanced Contributions we get: $\left(F_{v}(G)-F_{v}\left(G-\Gamma_{G}(u)\right)\right) / \omega(u)=0$ for every $u \in V$. It means that the centrality of $v$ does not depend on edges between other nodes; thus, it is the same as in graph with no edges: $F_{v}(G)=F_{v}(V, \emptyset, \omega, \mathbf{1})$. From Additivity, we have that $F_{v}(V, \emptyset, \omega, \mathbf{1})=F_{v}(V, \emptyset, \omega, \mathbf{1})+$ $F_{v}(V, \emptyset, \omega, \mathbf{1})=0$ for every node $v$, which implies $F_{v}(G)=0$.

(b) Since $v$ is a leaf, $\Gamma_{G}(v)=\{\{v, u\}\}$. From Additivity $F_{u}(G)-F_{u}\left(G-\Gamma_{G}(v)\right)=F_{u}\left(V, \Gamma_{G}(v), \omega, \mathbf{1}\right)$. In graph $G-\Gamma_{G}(u)$ node $v$ is isolated; thus, from (a): $F_{v}\left(G-\Gamma_{G}(u)\right)=0$. Combining these facts with Equation (3) and Weighted One-Edge Normalization, we get: $F_{v}(G) / \omega(u)=F_{u}\left(V, \Gamma_{G}(v), \omega, \mathbf{1}\right) / \omega(v)=1$.

(c) Assume $N_{G}(v)=\left\{u_{1}, u_{2}, \ldots, u_{k}\right\}$. The set of edges in the component of $v$ can be divided into the following $k$ sets: $E_{1}, E_{2}, \ldots, E_{k}$, such that $\left\{v, u_{i}\right\} \in E_{i}$, and $\bigcup E_{i} \cap \bigcup E_{j}=$ $\{v\}$ for every $i, j \in\{1, \ldots, k\}, i \neq j$. From Additivity: $F_{v}(G)=\sum_{1<i<k} F_{v}\left(V, E_{i}, \omega, \mathbf{1}\right)+F_{v}\left(G-\left(E_{1} \cup \ldots \cup E_{k}\right)\right)$. In each graph $\left(V, E_{i}, \omega, \mathbf{1}\right)$ node $v$ is a leaf and in graph $G-$ $\left(E_{1} \cup \ldots \cup E_{k}\right)$ node $v$ is isolated. Thus, from (a) and (b) we get that $F_{v}(G)=\sum_{u \in N_{G}(v)} \omega(u)$.

Building upon this result, we get an axiomatization of the Weighted Degree Centrality (for node-weighted graphs).

Theorem 5. If a centrality measure, F, satisfies Inverse Weighted Balanced Contributions, Additivity, Weighted OneEdge Normalization, Star-Max, Monotonicity, then for every node-weighted graph $G=(V, E, \omega, \mathbf{1})$ and $v \in V$ it is equal to the Weighted Degree Centrality: $F_{v}(G)=W D_{v}(G)$.

The proof, based on Theorem 4, uses the similar reasoning as the proof of Theorem 2 .

Let us focus on the Attachment Centrality and examine Gain-loss for weighted graphs in the following example.

Example 2. Let $V=\{v, u, w\}$ and consider two graphs: $G_{1}=(V,\{\{v, u\},\{v, w\}\}, \omega, \mathbf{1})$ and $G_{2}=$ $(V,\{\{v, u\},\{u, w\}\}, \omega, \mathbf{1})$. For these graphs we have:

$$
\begin{aligned}
& \sum_{v \in V} W D_{v}\left(G_{1}\right)=2 \cdot \omega(v)+\omega(u)+\omega(w), \\
& \sum_{v \in V} W D_{v}\left(G_{2}\right)=\omega(v)+2 \cdot \omega(u)+\omega(w) .
\end{aligned}
$$

Thus, $\sum_{v \in V} W D_{v}\left(G_{1}\right) \neq \sum_{v \in V} W D_{v}\left(G_{2}\right)$ if $\omega(v) \neq$ $\omega(u)$. In result, we know that there exists no measure such that $F_{v}(G)=W D_{v}(G)$ if $G$ is a tree and satisfies Gain-loss for node-weighted graphs, i.e., such that adding edges to a connected component does not affect the sum of centralities.

To cope with this problem we ask the question: how the weight of a node contributes to the sum of centralities? In the case of the Weighted Degree Centrality, as we saw in Example 2, the more central the node is, the bigger impact its weight has on the sum of centralities. In fact, we observe that the impact of the weight of a node is exactly proportional to its centrality. We formalize this notion in the following axiom, called Node-Weight Impact.

Node-Weight Impact: For every $G=(V, E, \omega, \mathbf{1})$, every $v \in V$ and every $\alpha \in \mathbb{R}$,

$$
\sum_{u \in V} F_{u}(G)=F_{v}(V, E, \mathbf{1}, \mathbf{1}) \cdot \alpha+\sum_{u \in V} F_{u}\left(V, E, \omega_{v}^{\omega(v)-\alpha}, \mathbf{1}\right) .
$$

To introduce the Weighted Attachment Centrality we use the Weighted Shapley Value, originally proposed by [Shapley, 
1953a] and studied by [Kalai and Samet, 1987]. For a game, $f$, and vector of weights, $\omega: V \rightarrow \mathbb{R}^{+}$, the Weighted Shapley Value is defined as follows:

$$
W S V_{v}^{\omega}(f)=\sum_{\pi \in \Omega(V)} \prod_{u \in V} \frac{\omega(u)}{\sum_{w \in S_{u}^{\pi}} \omega(w)}\left(f\left(S_{v}^{\pi}\right)-f\left(S_{v}^{\pi} \backslash\{v\}\right)\right) .
$$

The Weighted Shapley Value rewards elements with higher weights. However, as shown in Example 1, in our case we need an inverse notion. That is why, we will use Weighted Shapley Value, but with the inverse weight function $\frac{1}{\omega}$ defined as follows: $\frac{1}{\omega}(v)=\frac{1}{\omega(v)}$.

Definition 2. The Weighted Attachment Centrality for a node-weighted graph $G=(V, E, \omega, \mathbf{1})$ is defined as follows:

$$
W A_{v}(G)=W S V_{v}^{\frac{1}{\omega}}\left(f_{G}^{A}\right),
$$

where $f_{G}^{A}(S)=\sum_{v \in S} A_{v}(S, E[S], \mathbf{1}, \mathbf{1}) \cdot \omega(v)$.

Haeringer [1999] proposed the Weighted Myerson value, calculated as the Weighted Shapley value for restricted game $f / G$. The Attachment Centrality for node-weighted graphs can be considered as the Weighted Myerson value for inverse weight vector $\frac{1}{\omega}$ and game $f_{G}^{A}$.

In the following lemma, we prove that the Weighted Attachment Centrality is equal to the Attachment Centrality for unweighted graphs.

Lemma 6. For every unweighted graph $G=(V, E, \mathbf{1}, \mathbf{1})$, $W A_{v}(G)=A_{v}(G)$.

Proof. From Definition 2: $f_{G}^{A}(S)=\sum_{v \in S} A_{v}(S, E[S], \mathbf{1}, \mathbf{1})$. From Definition 1: $\sum_{v \in S} A_{v}(S, E[S], \mathbf{1}, \mathbf{1})=f_{G}^{*}(S)$. Thus, $f_{G}^{A}=f_{G}^{*}$. Moreover, $W S V_{v}^{1}(f)=S V_{v}(f)$ for every $f$ and we get that $W A_{v}(G)=W S V_{v}^{1}\left(f_{G}^{A}\right)=S V_{v}\left(f_{G}^{*}\right)=A_{v}(G)$.

In the main theorem of this section - Theorem 7 - we prove that by adding Node-Weight Impact to axioms from Theorem 3 we get a characterization of the Weighted Attachment Centrality. Note that we use Gain-loss for unweighted graphs, since it is undesirable for node-weighted graphs (see Example 2).

Theorem 7. If a centrality measure, $F$, satisfies Inverse Weighted Balanced Contributions, Additivity, Weighted OneEdge Normalization, Gain-loss for unweighted graphs and Node-Weight Impact, then for every node-weighted graph $G=(V, E, \omega, \mathbf{1})$ and $v \in V$ it is equal to the Weighted Attachment Centrality: $F_{v}(G)=W A_{v}(G)$.

Proof. Since Inverse Weighted Balanced Contributions, Additivity and Weighted One-Edge Normalization imply Balanced Contributions, Additivity and One-Edge Normalization when $\omega=1$, from Theorem 3 we have that $F_{v}(G)=A_{v}(G)$ for every unweighted graph $G$. Let $F, F^{\prime}$ be two centralities that satisfy Inverse Weighted Balanced Contributions, NodeWeight Impact, and Additivity and assume $F_{v}(G)=F_{v}^{\prime}(G)$ for every unweighted graph $G=(V, E, \mathbf{1}, \mathbf{1})$. To prove uniqueness, it remains to show that $F_{v}(G)=F_{v}^{\prime}(G)$ for every node-weighted graph $G=(V, E, \omega, \mathbf{1})$.

Fix graph $G=(V, E, \omega, \mathbf{1})$ and $u \in V$. From Node-Weight Impact we get: $\sum_{v \in V} F_{v}(G)=(\omega(u)-1) F_{v}(V, E, \mathbf{1}, \mathbf{1})+$ $\sum_{v \in V} F_{v}\left(V, E, \omega_{u}^{1}, \mathbf{1}\right)$. Using the same argument for graph $\left(V, E, \omega_{u}^{1}, \mathbf{1}\right)$ and sequentially for all other nodes we get: $\sum_{v \in V} F_{v}(G)=\sum_{v \in V} F_{v}(V, E, \mathbf{1}, \mathbf{1}) \cdot \omega(v)$. Thus, we get that $\sum_{v \in V} F_{v}(G)=\sum_{v \in V} F_{v}^{\prime}(G)$. Moreover, from Additivity we get $F_{v}(V, \emptyset, \omega, \mathbf{1})=0=F_{v}^{\prime}(V, \emptyset, \omega, \mathbf{1})$.

From [Skibski et al., 2017, Lemma 13] we know that there exists a unique centrality that satisfies Balanced Contributions and the sum of centralities in every graph as well as values in empty graph $(V, \emptyset)$ are specified. Using the same reasoning for weighted graphs and weighted version of Balanced Contributions we get that $F=F^{\prime}$.

It remains to prove that the Weighted Attachment Centrality satisfies all axioms. We consider them one by one:

- Weighted One-Edge Normalization: Consider graph $G=(V,\{\{v, u\}\}, \omega, \mathbf{1})$. From Definition $2 f_{G}^{A}(S)=$ $\omega(v)+\omega(u)$ if $v, u \in S$, and $f_{G}^{A}(S)=0$, otherwise. Thus, it is a unanimity game and from [Kalai and Samet, 1987] we get that $W A_{v}(G)=W S V_{v}^{\frac{1}{\omega}}\left(f_{G}^{A}\right)=$ $\frac{1}{\omega(v)} /\left(\frac{1}{\omega(v)}+\frac{1}{\omega(u)}\right) \cdot(\omega(v)+\omega(u))=\omega(u)$.

- Gain-loss for unweighted graphs: Immediate from Lemma 6 and [Skibski et al., 2016].

- Node-Weight Impact: From Definition 2 and the definition of Weighted Shapley value we get that $\sum_{v \in V} W A_{v}(G)=f_{G}^{A}(V)=\sum_{v \in V} A_{v}(V, E, \mathbf{1}, \mathbf{1})$. $\omega(v)$ for every graph $G=(V, E, \omega, \mathbf{1})$. This implies Node-Weight Impact.

- Additivity: Fix graph $G=(V, E, \omega, \mathbf{1})$, two sets $S, T \subseteq$ $V$ and $v \in V$, such that $S \cap T=\{v\}$. From the analysis of cut-vertices in [Skibski et al., 2016, Theorem 4] and the fact that the Attachment Centrality of an isolated node is zero, we get that $A_{u}(V, E[S], \mathbf{1}, \mathbf{1})+$ $A_{u}(V, E[T], \mathbf{1}, \mathbf{1})=A_{u}(V, E[S] \cup E[T], \mathbf{1}, \mathbf{1})$ for every $u \in V$. This combined with Definition 2 implies that for every $U \subseteq V: f_{(V, E[S], \omega, \mathbf{1})}^{A}(U)+f_{(V, E[T], \omega, \mathbf{1})}^{A}(U)=$ $f_{(V, E[S] \cup E[T], \omega, \mathbf{1})}^{A}(U)$. Since the Weighted Shapley value is additive we get $A V_{u}(V, E[S], \omega, \mathbf{1})+$ $A V_{u}(V, E[T], \omega, \mathbf{1})=A V_{u}(V, E[S] \cup E[T], \omega, \mathbf{1})$ for every $u \in V$ (including $u=v$ ).

- Inverse Weighted Balanced Contributions: Consider graph $G=(V, E, \omega, \mathbf{1})$. Fix node $v \in V$ and subset $S \subseteq$ $V$ such that $v \in S$. Consider graph $G^{\prime}=G-\Gamma_{G}(v)$. Since $v$ is isolated in $G^{\prime}$ we have $A_{v}\left(G^{\prime}[S]\right)=0$ and $A_{u}\left(G^{\prime}[S]\right)=A_{u}\left(G^{\prime}[S \backslash\{v\}]\right)=A_{u}(G[S \backslash\{v\}])$ for every $u \in V \backslash\{v\}$. Combined with Definition 2 we get

$$
f_{G-\Gamma_{G}(v)}^{A}(S)=f_{G}^{A}(S \backslash\{v\}) .
$$

From the formula of the Weighted Shapley value it is visible that for arbitrary game $g$, node $u \in V$ and game $g^{\prime}$ defined as follows: $g^{\prime}(S)=g(S)-g(S \backslash\{u\})$ we have $W S V_{u}^{\omega}(g)=W S V_{u}^{\omega}\left(g^{\prime}\right)$. Applying this observation and Equation (4) to left-hand side of the Inverse Weighted Balanced Contributions condition we get $\omega(u) \cdot\left(A V_{u}(G)-A V_{u}\left(G-\Gamma_{G}(v)\right)\right)=\omega(u)$. $W S V_{u}^{\frac{1}{\omega}}\left(f_{G}^{A}-f_{G-\Gamma_{G}(v)}^{A}-f_{G-\Gamma_{G}(u)}^{A}+f_{G-\left(\Gamma_{G}(v) \cup \Gamma_{G}(u)\right)}^{A}\right)$. In this formula, game is symmetric for $v$ and $u$, thus payoffs of nodes $v$ and $u$ are proportional to their weights.

This concludes the proof of Theorem 7 . 


\section{Node- and Edge-Weighted Graphs}

So far, we considered only weights of nodes. In this section, we show how weights of edges can be incorporated in the Weighted Attachment Centrality. Again, we begin with an example that helps us understand how weights of edges affect importance of nodes.

Example 3. Let $G=(V,\{e\}, \omega, \rho)$ be a graph with one edge $e=\{v, u\}$ and consider weight $\rho$ of edge e. First, assume $\rho(e) \in(0,1)$. In such case, weight can be interpreted as the probability that a given edge exists. Thus, with probability $\rho(e)$ edge exists $(G=(V,\{e\}, \omega, \mathbf{1}))$, and with probability $(1-\rho(e))$ edge does not exist $(G=(V, \emptyset, \omega, \mathbf{1}))$. This interpretation is consistent with the Weighted Degree Centrality:

$$
\begin{aligned}
& W D_{v}(V,\{e\}, \omega, \rho)=\rho(e) \cdot W D_{v}(V,\{e\}, \omega, \mathbf{1}) \\
& \quad+(1-\rho(e)) \cdot W D_{v}(V, \emptyset, \omega, \mathbf{1})=\rho(e) \cdot \omega(u) .
\end{aligned}
$$

Now, assume $\rho(e)=k$ for some $k \in \mathbb{N}$. This graph can be interpreted as the multigraph with the set of edges $E=$ $\{e, e, \ldots, e\},|E|=k$. By treating each edge separately the Weighted Degree Centrality equals: $W D_{v}(V,\{e\}, \omega, \rho)=$ $\rho(e) \cdot \omega(u)$. While both interpretations are based on different insights, they both characterize the same property: the profit from the edge is proportional to its weight.

Our analysis from Example 3 leads to the following axiom:

Edge-Weight Proportionality: Let $G=(V, E, \omega, \rho)$ be a graph. For every $v \in V, e \in E$ and $\alpha, \beta \in \mathbb{R}^{+}$,

$$
\begin{gathered}
\alpha \cdot\left(F_{v}\left(V, E, \omega, \rho_{e}^{\beta}\right)-F_{v}\left(V, E \backslash\{e\}, \omega, \rho_{-e}\right)\right)= \\
\beta \cdot\left(F_{v}\left(V, E, \omega, \rho_{e}^{\alpha}\right)-F_{v}\left(V, E \backslash\{e\}, \omega, \rho_{-e}\right)\right) .
\end{gathered}
$$

In the following theorem we show that if centrality satisfies Edge-Weight Proportionality, then it is uniquely defined by specifying values on graphs without edge-weights, i.e., formally, where weight of each edge equals 1 .

Theorem 8. If a centrality measure, $F$, satisfies Edge-Weight Proportionality, then for every graph $(V, E, \omega, \rho)$ :

$$
\begin{aligned}
& F_{v}(V, E, \omega, \rho)= \\
& \sum_{M \subseteq E}\left(\prod_{e \in M} \rho(e) \prod_{e \in E \backslash M}(1-\rho(e)) \cdot F_{v}(V, M, \omega, \mathbf{1})\right) .
\end{aligned}
$$

Sketch of Proof. For graph $G=(V, E, \omega, \rho)$, let us denote by $E_{1} \subseteq E$ the subset of edges with weight 1: $E_{1}=\{e \in$ $E: \rho(\bar{e})=1\}$. We will prove Equation (5) by induction on the size of set $E \backslash E_{1}$, i.e., the number of edges in a graph with the weight different than 1 . If $\left|E \backslash E_{1}\right|=0$, then $\rho=1$ and Equation (5) trivially holds. Assume Equation (5) is satisfied for every graph $G=(V, E, \omega, \rho)$ such that $\left|E \backslash E_{1}\right|<k$ for $k>0$. Now, consider $G=(V, E, \omega, \rho)$ such that $\left|E \backslash E_{1}\right|=k$. From Edge-Weight Proportionality for an arbitrary edge $e^{*} \in E \backslash E_{1}, \alpha=1$ and $\beta=\rho\left(e^{*}\right)$ we get $F_{v}(V, E, \omega, \rho)=\rho\left(e^{*}\right) \cdot F_{v}\left(V, E, \omega, \rho_{e^{*}}^{1}\right)+\left(1-\rho\left(e^{*}\right)\right)$. $F_{v}\left(V, E \backslash\left\{e^{*}\right\}, \omega, \rho_{-e^{*}}\right)$. Using inductive assumption we get Equation (5), which concludes the proof of Theorem 8.

Now, we are ready to define the Weighted Attachment Centrality for node- and edge-weighted graphs.
Definition 3. The Weighted Attachment Centrality for graph $G=(V, E, \omega, \rho)$ is defined as follows:

$$
W A_{v}(G)=W S V_{v}^{\frac{1}{\omega}}\left(f_{G}^{P}\right),
$$

where $f_{G}^{P}=\sum_{M \subseteq E} \prod_{e \in M} \rho(e) \prod_{e \in E \backslash M}(1-\rho(e)) f_{(V, M, \omega, \mathbf{1})}^{A}$.

For a graph $G=(V, E, \mathbf{1}, \rho)$ with unweighted nodes, the Weighted Attachment Centrality is equal to the Probabilistic Myerson value, defined by Calvo et al. [1999] with characteristic function $f_{G}^{*}$.

Based on Theorem 8, we get the following two axiomatizations of the Weighted Degree Centrality and the Weighted Attachment Centrality.

Theorem 9. There is a unique centrality measure that satisfies Inverse Weighted Balanced Contributions, Weighted OneEdge Normalization, Additivity, Star-Max, Monotonicity, and Edge-Weight Proportionality; this measure is the Weighted Degree Centrality.

Proof. From Theorem 5 and 8 it is enough to show that the Weighted Degree Centrality satisfies Edge-Weight Proportionality. This is straightforward from Equation (1).

Theorem 10. There is a unique centrality measure that satisfies Inverse Weighted Balanced Contributions, Weighted OneEdge Normalization, Additivity, Gain-loss for unweighted graphs, Node-Weight Impact and Edge-Weight Proportionality; this measure is the Weighted Attachment Centrality.

Proof. Immediate from Theorems 7 and 8.

\section{Related Work}

Amer and Giménez [2004] proposed the first measure of connectivity by computing semi-value of a game that assigns 1 if coalition is connected, and 0 otherwise. This solution works only for unweighted graphs. Lindelauf et al. [2013] expanded this concept to weighted graphs by using an arbitrary function if coalition is connected. The authors proposed several definitions of function $f$, but only for specific terrorist networks. Michalak et al. [2013] analysed the computational properties of these measures and proposed using the Myerson value instead of the Shapley value for connectivity games. Skibski et al. [2014] improved those algorithms and introduced a class of measures. However, no specific measures were proposed. Above concepts were only tested empirically and no axiomatic analysis was offered.

\section{Conclusions}

In this paper, we proposed Weighted Attachment Centrality an extension of the Attachment Centrality to node- and edgeweighted graphs - and provided its axiomatization. In future work, we are keen to study the computational properties of the Weighted Attachment Centrality and to extend the Attachment Centrality to directed graphs.

\section{Acknowledgments}

Jadwiga Sosnowska and Oskar Skibski were supported by the Foundation for Polish Science within the Homing programme (Project title: "Centrality Measures: from Theory to Applications"). 


\section{References}

[Amer and Giménez, 2004] Rafael Amer and José Miguel Giménez. A connectivity game for graphs. Mathematical Methods of Operations Research, 60(3):453-470, 2004.

[Bagler, 2008] Ganesh Bagler. Analysis of the airport network of india as a complex weighted network. Physica A: Statistical Mechanics and its Applications, 387(12):29722980, 2008.

[Belau, 2014] Julia Belau. Consequences of connection failure - centrality and the importance for cohesion. In Public Choice Society, 2014.

[Brandes and Erlebach, 2005] Ulrik Brandes and Thomas Erlebach. Network analysis: Methodological foundations. Springer-Verlag, 2005.

[Calvo et al., 1999] Emilio Calvo, Javier Lasaga, and Anne van den Nouweland. Values of games with probabilistic graphs. Mathematical Social Sciences, 37(1):79-95, 1999.

[Derrible, 2012] S. Derrible. Network centrality of metro systems. PLoS ONE, 7(7), 2012.

[Freeman, 1979] Linton C. Freeman. Centrality in social networks: Conceptual clarification. Social Networks, 1(3):215-239, 1979.

[Haeringer, 1999] Guillaume Haeringer. Weighted Myerson value. International Game Theory Review, 1(02):187-192, 1999.

[Heitzig et al., 2010] Jobst Heitzig, Norbert Marwan, Yong Zou, Jonathan F Donges, and Jürgen Kurths. Consistently weighted measures for complex network topologies. Europ. Phys. J. B, 85:1-16, 2010.

[Kalai and Samet, 1987] Ehud Kalai and Dov Samet. On weighted Shapley values. International Journal of Game Theory, 16(3):205-222, 1987.

[Koschade, 2006] Stuart Koschade. A social network analysis of Jemaah Islamiyah: The applications to counterterrorism and intelligence. Studies in Conflict \& Terrorism, 29(6):559-575, 2006.

[Lindelauf et al., 2013] Roy Lindelauf, Herbert Hamers, and Bart Husslage. Cooperative game theoretic centrality analysis of terrorist networks: The cases of Jemaah Islamiyah and Al Qaeda. European Journal of Operational Research, 229(1):230-238, 2013.

[Michalak et al., 2013] Tomasz P. Michalak, Talal Rahwan, Piotr L. Szczepański, Oskar Skibski, Ramasuri Narayanam, Michael Wooldridge, and Nicholas R. Jennings. Computational analysis of connectivity games with applications to the investigation of terrorist networks. In Proceedings of 23rd International Joint Conference on Artificial Intelligence (IJCAI), pages 293-301, 2013.

[Moretti et al., 2010] Stefano Moretti, Vito Fragnelli, Fioravante Patrone, and Stefano Bonassi. Using coalitional games on biological networks to measure centrality and power of genes. Bioinformatics, 26(21):2721-2730, 2010.
[Myerson, 1977] Roger B. Myerson. Graphs and cooperation in games. Mathematical Methods of Operations Research, 2(3):225-229, 1977.

[Myerson, 1980] Roger B. Myerson. Conference structures and fair allocation rules. International Journal of Game Theory, 9:169-82, 1980.

[Narayanam et al., 2014] Ramasuri Narayanam, Oskar Skibski, Hemank Lamba, and Tomasz P. Michalak. A Shapley value-based approach to determine gatekeepers in social networks with applications. In Proceedings of 21st European Conference on Artificial Intelligence (ECAI), pages 651-656, 2014.

[Nepusz et al., 2012] Tamás Nepusz, Haiyuan Yu, and Alberto Paccanaro. Detecting overlapping protein complexes in protein-protein interaction networks. Nature methods, 9(5):471-472, 2012.

[Opsahl et al., 2010] Tore Opsahl, Filip Agneessens, and John Skvoretz. Node centrality in weighted networks: Generalizing degree and shortest paths. Social networks, 32(3):245-251, 2010.

[Palla et al., 2007] Gergely Palla, Albert-László Barabási, and Tamás Vicsek. Quantifying social group evolution. Nature, 446(7136):664-667, 2007.

[Shapley, 1953a] Lloyd S Shapley. Additive and nonadditive set functions. Princeton University, 1953.

[Shapley, 1953b] Lloyd S. Shapley. A value for n-person games. In H.W. Kuhn and A.W. Tucker, editors, Contributions to the Theory of Games, volume II, volume II, pages 307-317. Princeton University Press, 1953.

[Skibski et al., 2014] Oskar Skibski, Tomasz P. Michalak, Talal Rahwan, and Michael Wooldridge. Algorithms for the Shapley and Myerson values in graph-restricted games. In Proceedings of 13th International Conference on Autonomous Agents and Multiagent Systems (AAMAS), pages 197-204, 2014.

[Skibski et al., 2016] Oskar Skibski, Talal Rahwan, Tomasz P. Michalak, and Makoto Yokoo. Attachment centrality: An axiomatic approach to connectivity in networks. In Proceedings of 15th International Conference on Autonomous Agents and Multiagent Systems (AAMAS), pages 168-176, 2016.

[Skibski et al., 2017] Oskar Skibski, Tomasz P. Michalak, and Talal Rahwan. Axiomatic characterization of gametheoretic network centralities. In Proceedings of 31 st AAAI Conference on Artificial Intelligence (AAAI), pages 698705, 2017.

[Tarkowski et al., 2016] Mateusz K Tarkowski, Piotr Szczepański, Talal Rahwan, Tomasz P. Michalak, and Michael Wooldridge. Closeness centrality for networks with overlapping community structure. In Proceedings of 30th AAAI Conference on Artificial Intelligence (AAAI), pages 622-629, 2016. 